\title{
A MAGNETIC MODEL OF THE CHROMOSPHERE-CORONA TRANSITION REGION
}

\author{
A. H. GABRIEL \\ Astrophysics Research Division of the Appleton Laboratory, Culham Laboratory, \\ Abingdon, Berkshire, England
}

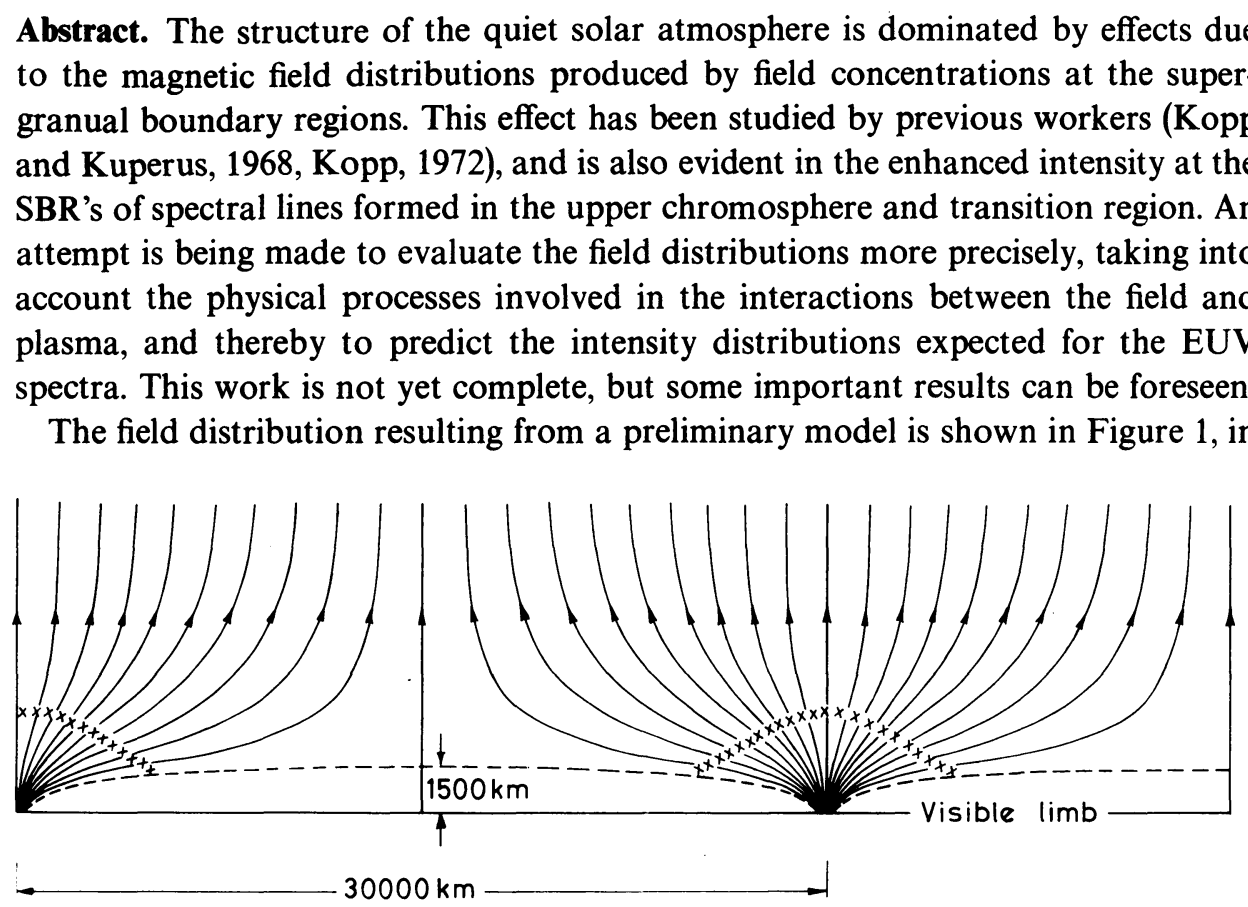

Fig. 1. A preliminary model for the supergranule structure, showing the primary ( $x$ 's) and secondary (dashed) transition regions.

which a mean field of $1 \mathrm{G}$ has been assumed. The lower boundary is the photospheric surface, and the dashed line is the upper limit to the velocity field characterising the super-granule convection flow. Two quite separate transition regions are produced. The first, indicated in the figure by $x$ 's is physically similar to that predicted by spherical solar models. It will be rather thicker than that arising in the spherical models, and similar to that proposed by Kopp (1972). It is from this region that the majority of the EUV radiation for transition ions will be emitted and it is therefore termed the 'primary transition region'. The dashed surface covering the network centre is also a transition region in the sense that it separates coronal from chromospheric material. However, it is closely parallel to the magnetic field, and in this simple model will have an infinite temperature gradient, i.e. negligible material at 
transition temperatures. This is referred to as the 'secondary transition region'. Obviously such a gradient will suffer a range of instabilities, which will result in a small mixing, and therefore a limited emission from a very thin transition layer. All these features are broadly consistent with the present EUV observations. One important consequence of the model shown in Figure 1 is that the secondary transition region will be at a higher pressure (and density) than the primary region. This is the opposite to the situation found in other cases of inhomogeneities, such as quiet to active region comparisons, in which the higher densities are normally associated with higher brightness regions. Spectroscopic observations of the network using density sensitive line ratios could therefore prove an important test for the validity of this model.

\section{References}

Kopp, R. A.: 1972, Solar Phys. 27, 373.

Kopp, R. A. and Kuperus, M.: 1968, Solar Phys. 4, 212.

\section{DISCUSSION}

Athay: It seems to be generally assumed that the supergranulation circulation extends through the chromosphere. The only actual observation of the supergranule motion however is restricted to the photosphere. The question I would like to ask is whether the observers that are here see any prospect of measuring the supergranulation circulation in the chromosphere?

Deubner: Actually the dependence of supergranule circulation has been measured in several spectral lines. It shows a slight decrease from photospheric lines up to lines such as the sodium D lines, but I am not sure whether it has been measured in $\mathrm{H} \alpha$.

Gabriel: If the chromosphere is not circulating at these heights, then the field will spread out much more rapidly, and on this model you would expect radiation from the transition region lines coming right down to the photosphere.

Sturrock: Have you considered the effect of the field lines on the acoustic wave propagation and excitation as well as on the heat conduction?

Gabriel: Only to the extent that the heat conduction will be channelled along the magnetic field lines.

Sturrock: If acoustic waves are being produced in a magnetic field that is strongly inclined to the vertical, then the cut-off frequency is reduced and this could mean that much more of the turbulent energy is coupled into the acoustic waves as a result of an inclined field.

Giovanelli: I am still worried about this particular model because I do not think that it lines up with the things we see in the chromosphere. We do see fibrils that leave the network and turn over towards the horizontal, and these represent bundles that are discrete; they are not touching one another, at least not always. It doesn't seem to me that this particular model in which the field lines end up going vertically, is quite compatible with the picture we get from the calcium and hydrogen chromospheres. There the tubes seem to be two, three or even four thousand kilometers wide. I think that these need to be contained by an external gas pressure in which the field is lower. Your model shows all of the field lines swinging up and becoming vertical so that if you get to $10000 \mathrm{~km}$ or less, or even at 3000 or $4000 \mathrm{~km}$, the field scarcely knows what is down below. The model one gets from the chromosphere suggests that as the flux tubes come up they are kept separate.

. Gabriel: One limitation of this model is that it is two-dimensional, and I have assumed that the boundary of the network has a uniform field distribution whereas my understanding from the observations is that the field is fairly well clumped into bundles. That is the sort of thing that could provide the limitation you are describing.

Schmidt: I want to comment on this question. I think one is dealing with the quiet chromosphere where all of the flux is leaving the Sun, most probably to a very high percentage, is connected to the solar wind. I do not believe that fibrils are to be seen in regions of the Sun where the flux is connected to another supergranular boundary or another distant field concentration. If the fibrils connect to foot points 
then it is not a matter of the dimensionality of the model, but the model which we have for the fibrils embedded in that medium described by Dr Gabriel at greater heights. The height of the fibrils is probably something like $4000 \mathrm{~km}$ and they should be embedded in coronal matter which has field lines connected to the source regions described in this paper to the corona above the transition layer. This model is provided by Pikel'ner and I think is a beautiful non-LTE model.

Athay: I understand from talking with Jack Harvey at Kitt Peak Observatory that although the network is statistically embedded in a region of uniform polarity, it is also a common feature of the network to have small fields of opposite polarity adjacent to the main fields of the network. In other words the uniform field polarity of the network is a statistical result and does not hold in detail, but rather there is a substantial complement of opposite polarity present on a fine scale. Could you comment on the effect that this would have on the model that you were talking about?

Gabriel: I am not familiar with the work by Harvey but it would clearly introduce additional closed field lines. Isn't this very much in line with what Professor Schmidt just said?

Beckers: When we study the variation of spicule diameters with height, we find that spicules actually grow smaller when they go away from the limb. If spicules occurred in a divergent magnetic field configuration as you have drawn, I would expect the spicule diameter to increase with height, but that is not the case. Maybe this is related to the bundling that Giovanelli has mentioned, or maybe it is related to the possibility that spicules occur in voids in the magnetic field where the field lines have been pushed apart by the granulation, to form a region of avoidance that converges back to uniform field at greater heights. I didn't mention this yesterday but there is some evidence in the work I have done that this indeed may be the case - that the spicules are not on top of the filigree but open spaces between the filigree.

Vrabec: I would like to reply to Athay's comment concerning Jack Harvey's observations. I am not familiar with this work but our experience at Aerospace in looking at our K-line spectroheliograms is that for very extended regions of the network outside of active regions, the polarities seem to be very strictly of one magnetic polarity. The only place we observe a mixture of polarities is where these extended regions interface or in the active regions themselves.

Schmidt: May I just at this point stress the terrible importance of such observations, of stating just that there are no opposite polarities intermingled, or there are. This deserves the utmost effort. As soon as there are opposite polarities within an average quiet chromosphere cell, even though it may be only on a very small scale, the models of Pikel'ner and Uchida for spicules become very efficient and could easily describe what we see. But please don't find spurious opposite polarities.

Acton: At those locations where we do see two polarities in otherwise quiet regions, this is precisely where in the corona we see X-ray bright points. Up to this point there is a one-to-one correlation that wherever you see a bipolar structure, no matter how small, there is a bright point in the corona. When you look at high-resolution photographs from the corona compared to chromospheric or transition region structure, the corona is generally very diffuse, except at these small bipolar features. Clearly when the bipolar features are present the model Dr Gabriel has been discussing breaks down and we have an entirely different state of affairs.

Brueckner: Dr Gabriel referred to the observations of NRL, and I would like to show slides in order to make sure that everyone understands just what has been observed. These are mixed slides from Skylab and recent rocket observations. The network in helium 584 has a good resemblance to the $\mathrm{H}$ and $\mathrm{K}$ network. The network structure disappears at the polar caps as can be seen in the slide. However, bright points are still visible in the polar cap region and we identify these with bipolar regions. The network in HeII, 304 is identical to the network in HeI. It is a carbon copy, including the absence of network in the polar caps. In OIV your first impression is that it is similar to the helium network, but on closer inspection there is more and more concentration into bright points and there is no region of avoidance in the polar caps, at either pole. In OVI there is even more and more of a concentration into very bright points, and I am sure they are below the resolution of the instrument in size. In Ne vil almost everything is concentrated in the very bright points that are below the resolution limits of the instrument in size. The polar cap is visible but very difficult to make out. The last slide shows a comparison of a helium image with an image of the corona. It is obvious from the X-ray picture of the corona that the coronal holes are very depressed. You find a close correlation between the absence of the helium network and the corona, but you also find that the bright points that still exist can be traced back into the corona. You also find that at the boundary of the suppressed region where you see coronal rays coming out, you can see that the helium emission is enhanced.

Vrabec: I think we have an important question for discussion. Dr Acton has just mentioned that wherever they see bipolar regions they see bright points in the corona. Now Brueckner has shown us 
pictures indicating that there are bright points in the polar cap regions and there is the interference that these overlie mixed-polarity regions. But the rest of the Sun is completely dotted with more bright points. Is the inference that all of these bright points are bipolar? That appears to be a real problem.

Jordan: Some years ago Tony Hearn and I did calculations where we allowed matter to flow out from the Sun as observed in the solar wind, and we calculated what effect this would have on the line emissions. The ions moved through the transition region with velocities consistent with the solar wind. The interesting result that we found, which we never got around to publishing, is that the HeIl line is very dependent upon the velocity because of the high excitation potential of the ions compared to the surrounding temperature. If you concentrate the solar wind motion into one-thirtieth of the solar area, you can raise the intensity of the $\mathbf{3 0 4}$ line by a factor of ten over the value you get for the static Sun. This could mean that the absence of network in HeII indicates an absence of outflow in the helium material. This effect would not be seen in any of the lines except the HeII lines and the HeI lines. It is not present in Ly- $\alpha$ because of the transfer problems. 